\title{
The luminosity function evolution of soft X-ray-selected active galactic nuclei in the RIXOS survey
}

\author{
M. J. Page, ${ }^{1}$ F. J. Carrera, ${ }^{1}$ G. Hasinger,${ }^{2}$ K. O. Mason, ${ }^{1}$ R. G. McMahon, ${ }^{3}$ \\ J. P. D. Mittaz, ${ }^{1}$ X. Barcons, ${ }^{4}$ R. Carballo, ${ }^{4,5}$ I. González-Serrano ${ }^{4}$ and \\ I. Pérez-Fournon ${ }^{6}$
}

\author{
${ }^{1}$ Mullard Space Science Laboratory, University College London, Holmbury St Mary, Dorking, Surrey RH5 6 NT \\ ${ }^{2}$ Astrophysikalisches Institut Potsdam, An der Sternwarte 16, Potsdam, Germany \\ ${ }^{3}$ Institute of Astronomy, Madingley Road, Cambridge CB3 OHA \\ ${ }^{4}$ Instituto de Fisica de Cantabria, CSIC-Universidad de Cantabria, 39005 Santander, Spain \\ ${ }^{5}$ Dept Fisica Moderna, Universidad de Cantabria, 39005 Santander, Spain \\ ${ }^{6}$ Instituto de Astrofisica de Canarias, C/Vía Láctea, 38200 La Laguna, Tenerife, Spain
}

Accepted 1996 February 16. Received 1996 January 22; in original form 1995 August 2

\begin{abstract}
A BSTRACT
A sample of 198 soft X-ray-selected active galactic nuclei (AGN) from the ROSAT International X-ray Optical Survey (RIXOS) is used to investigate the X-ray luminosity function and its evolution. RIXOS, with a flux limit of $3 \times 10^{-14} \mathrm{erg} \mathrm{s}^{-1}$ $\mathrm{cm}^{-2}(0.5$ to $2.0 \mathrm{keV})$, samples a broad range in redshift over $20 \mathrm{deg}^{2}$ of sky, and is almost completely identified; it is used in combination wtih the Einstein Extended Medium Sensitivity Survey (EMSS) to give a total sample of over $600 \mathrm{AGN}$. We find the evolution of AGN with redshift to be consistent with pure luminosity evolution (PLE) models in which the evolution slows markedly or stops at high redshifts $(z>1.8)$. We find that this result is not affected by the inclusion, or exclusion, of narrow-emission-line galaxies at low redshift in the RIXOS and EMSS samples, and is insensitive to uncertainties in the conversion between flux values measured with ROSAT and Einstein. We confirm, using a model-independent $\left\langle V_{\mathrm{e}} / V_{\mathrm{a}}\right\rangle$ test, that our survey is consistent with no evolution at high redshifts.
\end{abstract}

Key words: surveys - galaxies: active - galaxies: luminosity function, mass function cosmology: observations - X-rays: galaxies.

\section{INTRODUCTION}

$\mathrm{X}$-ray properties are becoming an increasingly important tool for selecting samples of AGN, and sample sizes are approaching that of ultraviolet-excess (UVX) selected optical samples.

The largest currently available sample of serendipitous $\mathrm{X}$-ray-selected AGN comes from the Einstein Extended Medium Sensitivity Survey (EMSS, see Stocke et al. 1991), with a sky coverage of $778 \mathrm{deg}^{2}$. However, the EMSS is dominated by low-redshift objects (median $z \sim 0.2$ ) because of its high limiting flux (typically $>10^{-13} \mathrm{erg} \mathrm{s}^{-1} \mathrm{~cm}^{-2}$ in the energy range 0.5 to $2.0 \mathrm{keV}$ ). Deeper Einstein surveys (Primini et al. 1991) identified only 11 AGN. With the arrival of ROSAT the possibilities for deeper surveys have been realized: the Cambridge-Cambridge ROSAT Serendipity
Survey covers about $4 \mathrm{deg}^{2}$ and has a flux limit of $2 \times 10^{-14}$ $\mathrm{erg} \mathrm{s}^{-1} \mathrm{~cm}^{-2}$ from 0.5 to $2.0 \mathrm{keV}$ (see Boyle et al. 1995); the survey of Boyle et al. (1994) contains 107 broad-line AGN and probes to flux levels lower than $4 \times 10^{-15} \mathrm{erg} \mathrm{s}^{-1} \mathrm{~cm}^{-2}$ ( 0.5 to $2.0 \mathrm{keV}$ ), but is only 70 per cent complete at this flux limit and covers an area of less than $1.5 \mathrm{deg}^{2}$ in total. This survey is almost devoid of low-redshift objects, with only three AGN of $z<0.4$, and median $z=1.5$. Even deeper surveys, with even smaller sky coverage (Hasinger et al. 1993; Branduardi-Raymont et al. 1991), are still in the process of optical identification.

The ROSAT International X-ray Optical Survey (hereafter RIXOS, see Mason et al., in preparation) occupies a position between the EMSS and the deeper ROSAT surveys, with a sky coverage of over $20 \mathrm{deg}^{2}$ and a limiting flux of $3 \times 10^{-14} \mathrm{erg} \mathrm{s}^{-1} \mathrm{~cm}^{-2}(0.5$ to $2.0 \mathrm{keV})$. It is constructed 
from 81 long ( $>8000 \mathrm{~s}$ ) ROSAT pointings made with the Position Sensitive Proportional Counter (PSPC) at the focus of the X-ray telescope. The RIXOS AGN sample consists of 198 objects, the largest ROSAT-selected AGN sample to date, and is particularly useful for investigating evolution models because of its broad sampling of redshifts.

In this paper we present the results of an analysis of the differential X-ray luminosity function (XLF) and its evolution with redshift, using the RIXOS sample. In Section 2 we describe briefly the RIXOS survey and present details of the RIXOS AGN sample, while in Section 3 we discuss the $\log N-\log S$ relation of the RIXOS AGN compared with that of the EMSS AGN. In Section 4 the methods used in this analysis are explained and in Section 5 we present our results, which are discussed in Section 6. Our conclusions are presented in Section 7.

Throughout this paper a Friedmann model universe has been assumed, and a value of $50 \mathrm{~km} \mathrm{~s}^{-1} \mathrm{Mpc}^{-1}$ has been adopted for the Hubble constant $H_{0}$; two different values for the deceleration parameter, $q_{0}=0$ and 0.5 , have been used.

\section{THE RIXOS SAMPLE OF AGN}

\subsection{Observations and data reduction}

The X-ray sources in RIXOS were found in a total of 81 ROSAT PSPC pointings. The target of each observation and a small region around it has been excluded from the analysis, so that RIXOS consists entirely of serendipitously discovered sources. Sources more than 17 arcmin off-axis have also been excluded because of their larger positional uncertainty and possible masking by the detector window support structure. Furthermore, only sources detected in the harder ROSAT energy band $(0.4$ to $2.4 \mathrm{keV})$ are included; the poorer point spread function, interstellar absorption, diffuse Galactic X-ray emission, and the increased contribution of Galactic stars complicate the detection of extragalactic sources in the 0.1 - to $0.4-\mathrm{keV}$ band. Using finding charts from the automatic plate measuring (APM) facility at the Royal Greenwich Observatory, Cambridge, optical spectra were taken for all optical counterparts within the $1 \sigma$ error circle of each X-ray source. If no likely counterpart was found, the optical counterparts in the larger $2 \sigma$ and $3 \sigma$ error circles were investigated. Where the APM finding charts were not sufficient (for example, if no optical counterparts appeared near the X-ray source position), CCD images were obtained using the Nordic Optical Telescope (NOT), or the Isaac Newton Group of telescopes (ING) on La Palma. Full details of the optical imaging and spectroscopic observations and data reduction are given in Mason et al. (in preparation).

In this analysis, the term $\mathrm{AGN}$ is used to refer to approximately the same range of objects as in Maccacaro et al. (1991), that is, objects with at least one broad $(>1000 \mathrm{~km}$ $\mathrm{s}^{-1}$ ) emission line and/or [O $\left.\mathrm{III}\right] 5007>\left[\mathrm{O}_{\mathrm{II}}\right]$ 3727. Hence the RIXOS AGN sample does include some narrow-line objects. These criteria have been deliberately chosen to avoid any significant difference between the RIXOS and EMSS optical selections, allowing the two samples to be meaningfully compared and combined. The effect of excluding narrow-line objects is discussed in Section 6.

\subsection{Construction of the AGN sample used in this analysis}

A limiting X-ray flux of $3.0 \times 10^{-14} \mathrm{erg} \mathrm{s}^{-1} \mathrm{~cm}^{-2}(0.5$ to $2.0 \mathrm{keV}$ ) was chosen for RIXOS, well above the detection threshold for all the ROSAT fields used. Owing to the constraints of optical telescope scheduling, some X-ray sources remain unobserved and/or unidentified. In the interests of keeping incompleteness and optical selection effects to a minimum, the entire RIXOS survey has not been used. In 62 of the 81 RIXOS fields, all objects have been identified or observed spectroscopically to the intended flux limit of $3.0 \times 10^{-14} \mathrm{erg} \mathrm{s}^{-1} \mathrm{~cm}^{-2}(0.5$ to $2.0 \mathrm{keV})$, while in each of the other 19 fields some, but not all, of the X-ray sources have been observed to this limit; these 19 fields are, however, fully observed to a flux of $8.4 \times 10^{-14} \mathrm{erg} \mathrm{s}^{-1} \mathrm{~cm}^{-2}(0.5$ to $2.0 \mathrm{keV}$ ) and are included in the RIXOS AGN sample with this flux limit. The overall spectroscopic completeness of the fields used in the RIXOS AGN sample is 93 per cent; the remaining 7 per cent of sources that are unidentified are those for which the optical counterpart(s) were too faint for us to obtain reliable optical spectra. We have made the assumption that the fraction of unidentified sources that are AGN is the same as that for the identified sources. Accordingly, the sky area used for this analysis has been corrected, in a similar fashion to that of Boyle et al. (1994), by multiplying the area by the fraction of sources identified; as the unidentified fraction is small, this has only a small effect on our results. Since optical completeness is a function of flux limit, we have calculated the effective sky area at $3.0 \times 10^{-14} \mathrm{erg} \mathrm{s}^{-1} \mathrm{~cm}^{-2}, 8.4 \times 10^{-14} \mathrm{erg} \mathrm{s}^{-1} \mathrm{~cm}^{-2}$, and three intermediate fluxes corresponding to significant changes in spectroscopic completeness. Again, the high level of completeness in RIXOS makes this a small correction, which has only a small effect on our results. The number of ROS $A T$ fields, corrected sky coverages and identified fractions at their respective limiting fluxes are listed in Table 1.

The redshift distribution, $N(z)$, of the RIXOS AGN sample is shown in Fig. 1. The sample has a significantly higher median redshift, 0.6, than the EMSS (0.2).

Table 1. RIXOS cumulative sky coverage corrected for incompleteness.

\begin{tabular}{|c|c|c|c|}
\hline Flux Limit & Corrected & Optically & Number of \\
\hline $\begin{array}{c}\left(\operatorname{erg~s}^{-1} \mathrm{~cm}^{-2}\right) \\
0.5-2 \mathrm{keV}\end{array}$ & $\begin{array}{l}\text { Area } \\
\left(\operatorname{deg}^{2}\right)\end{array}$ & $\begin{array}{l}\text { ldentitied } \\
\text { Fraction }\end{array}$ & $\mathrm{ds}$ \\
\hline
\end{tabular}

$\begin{array}{llll}3.0 \times 10^{-14} & 14.16 & 93 \% & 62 \\ 3.5 \times 10^{-14} & 14.36 & 95 \% & 62 \\ 5.0 \times 10^{-14} & 14.73 & 97 \% & 62 \\ 6.0 \times 10^{-14} & 15.09 & 99 \% & 62 \\ 8.4 \times 10^{-14} & 20.04 & 99 \% & 81\end{array}$




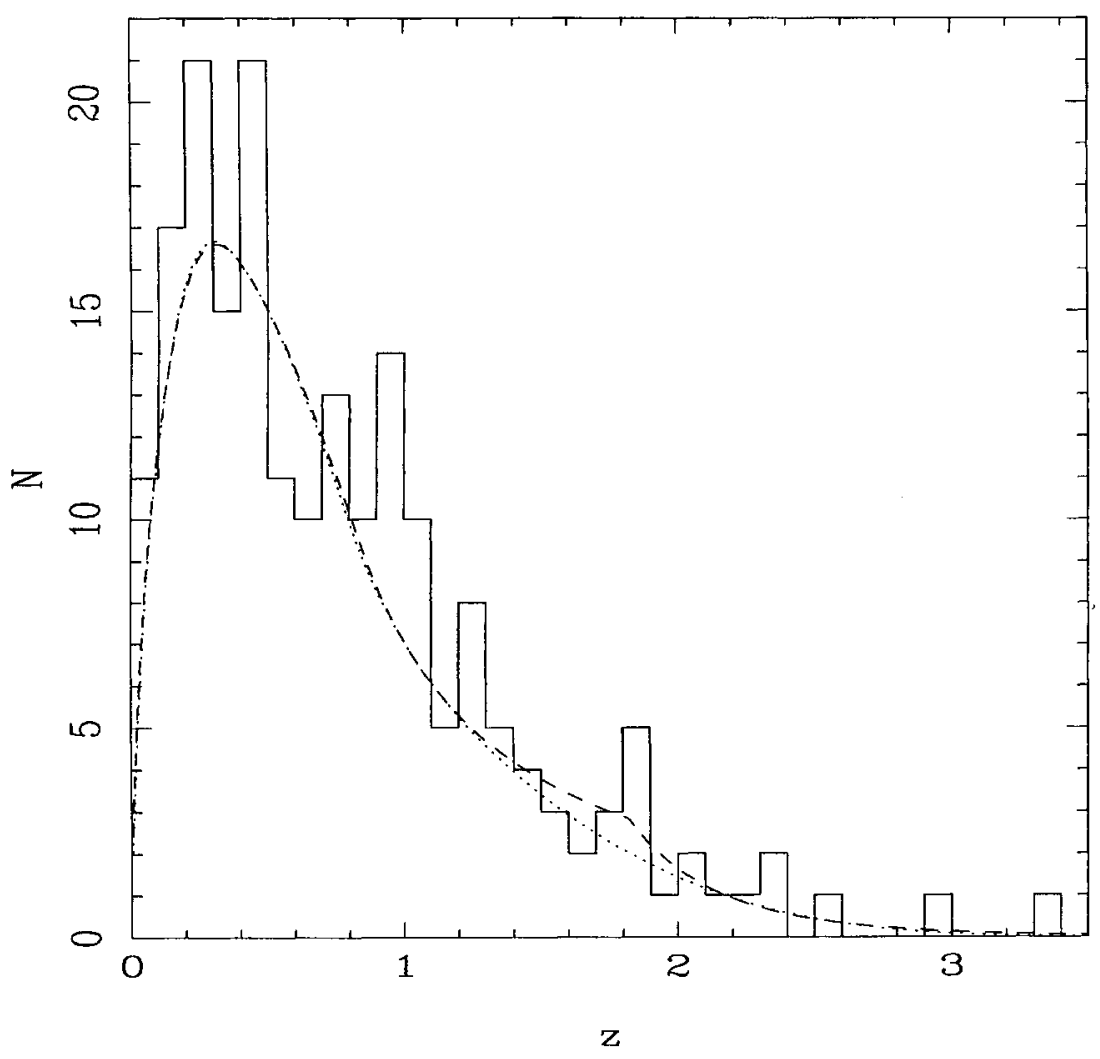

Figure 1. Redshift distribution, $N(z)$, of RIXOS. The solid histogram is the actual distribution, while the dashed and dotted lines are the $N(z)$ relations predicted by the power law with redshift cut-off and polynomial models respectively, for $q_{0}=0$.

To obtain the largest possible working sample of AGN, the RIXOS and EMSS surveys have been combined coherently (Avni \& Bahcall 1980) to give a total of over 600 AGN. This combined sample will be referred to as 'RIXOS + EMSS' hereafter. To correct the EMSS sample for incompleteness, the EMSS 'expected' AGN (see Maccacaro et al. 1991) have been included in the EMSS and EMSS + RIXOS samples. Throughout this analysis, a power-law X-ray spectrum has been assumed, $f_{v} \propto v^{-\alpha_{X}}$. For comparison of the results presented in this paper with those of Boyle et al. (1994) and Maccacaro et al. (1991), the analysis has been performed using $\alpha_{\mathrm{X}}=1$. The first results from X-ray colour analysis (see Mittaz et al., in preparation) indicate that this is quite representative of the RIXOS AGN, which have a median $\alpha_{x} \sim 1.01$. The scatter of $\alpha_{X}$ around the value 1.0 appears to be larger in the RIXOS sample than was found by Macaccaro et al. (1988) for EMSS AGN, although the hardening of source spectra with redshift (see Francis 1993) does not appear to be significant in the RIXOS sample and has not been included in this analysis. The X-ray luminosity-redshift $\left(L_{\mathrm{X}}, z\right)$ distributions for the RIXOS and EMSS AGN are compared in Fig. 2. As expected from a deeper survey, the RIXOS AGN typically have lower luminosity and/or higher redshift than the EMSS AGN.

\section{LOGN-LOGS}

The survey of Boyle et al. (1994) contains a larger number of AGN than would be predicted by extrapolating the
EMSS $\log N-\log S$ relation to lower fluxes; these authors suggested that this may be partly due to an error in their conversion from ROSAT to Einstein fluxes. In practice there are a number of factors that might affect the relative source counts and/or fluxes of the two samples, including differences in the source detection and parametrization algorithms, uncertainties in the detector response matrices used to transform count rates to fluxes, uncertainties in the spectral form used to convert from fluxes in the Einstein $0.3-3.5 \mathrm{keV}$ band to the ROSAT $0.5-2.0 \mathrm{keV}$ band, and the effects of incompleteness or other biases.

To assess these effects, Fig. 3 compares the integral $\log N-\log S$ relations for the RIXOS and EMSS AGN. This plot is derived using a conversion factor (CF) of 1.8 between the fluxes in the two bands which is appropriate for a powerlaw spectrum of $\alpha_{\mathrm{x}}=1$ if we use the standard published response matrices for ROSAT and Einstein. It is clear that RIXOS has a larger number of sources than the EMSS by about 30 per cent at almost all fluxes when using this conversion. Although RIXOS samples a larger area than the EMSS at low fluxes, there is significant overlap in the fluxes of objects found in the two samples, and there is thus no physical reason why the $\log N-\log S$ relations of the two surveys should be different.

Further investigation of the reasons for this discrepancy is beyond the scope of this paper. Instead we adopt an approach whereby we parametrize the discrepancy empirically, and investigate to what extent the uncertainty in this number affects the results on AGN evolution when we combine the RIXOS and EMSS samples. The method that we 


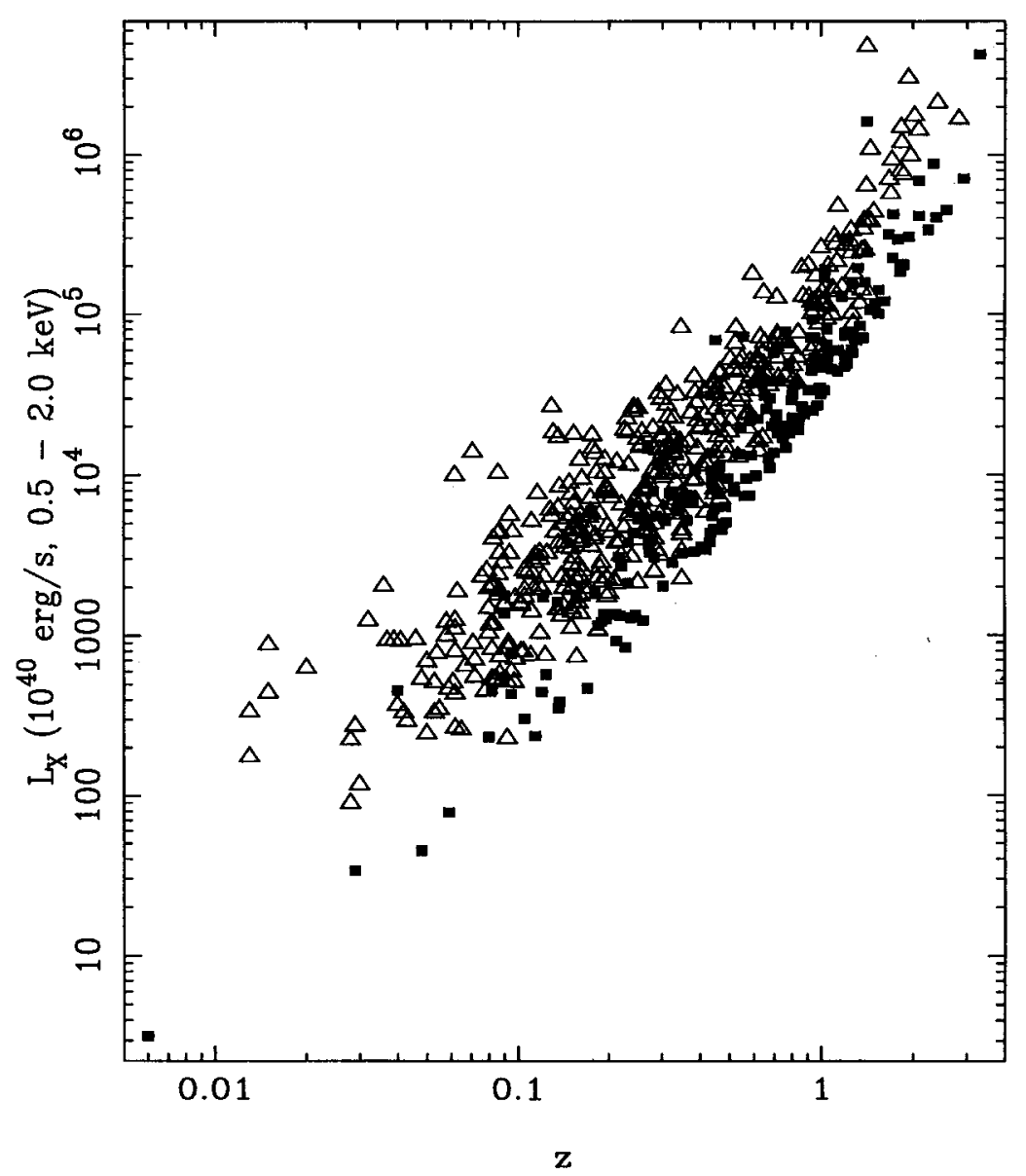

Figure 2. X-ray luminosity and redshift of RIXOS AGN (filled squares) and EMSS AGN (open triangles). Luminosities have been calculated using $q_{0}=0$.

use for this empirical parametrization is to find the CF for which the two $\log N-\log S$ relations are consistent. At the flux limits of the EMSS and RIXOS the $N(S)$ relation is well fitted by a power law. Using maximum likelihood, the two samples have been fitted simultaneously with a single power-law slope but different normalizations:

$\frac{\mathrm{d} N}{\mathrm{~d} S}=k_{\mathrm{E}} S^{-\gamma} \quad$ EMSS objects, $S(0.3-3.5 \mathrm{keV})$,

$\frac{\mathrm{d} N}{\mathrm{~d} S}=k_{\mathrm{R}} S^{-\gamma} \quad$ RIXOS objects, $S(0.5-2.0 \mathrm{keV})$.

There are two free parameters in this fit: the power-law slope $\gamma$ and the difference between the two normalizations $\left(k_{\mathrm{E}} / k_{\mathrm{R}}\right)$; the actual normalizations are found by requiring that the number of objects predicted by the $\log N-\log S$ relation for the sky coverage of RIXOS plus the sky coverage of the EMSS be equal to the total in RIXOS plus the total in the EMSS. The normalization difference should be related simply to the empirical CF from ROSAT to Einstein fluxes by

$\mathrm{CF}=\left(k_{\mathrm{E}} / k_{\mathrm{R}}\right)^{1 /(\gamma-1)}$.
We have assumed upper flux limits of $2 \times 10^{-11} \mathrm{erg} \mathrm{s}^{-1} \mathrm{~cm}^{-2}$ ( 0.3 to $3.5 \mathrm{keV})$ and $10^{-12} \mathrm{erg} \mathrm{s}^{-1} \mathrm{~cm}^{-2}(0.5$ to $2.0 \mathrm{keV})$ for the EMSS and RIXOS respectively, to reflect the selection against very bright sources in these surveys. This has only a small effect on the results; changing the upper flux limits to any reasonable value (or removing them) changes the best fit by only a fraction of the $1 \sigma$ statistical errors quoted below. The best-fitting slope $\gamma$ is found to be 2.56 , consistent with the slopes found when the two samples are fitted independently $(2.61 \pm 0.06$ for the EMSS and $2.45 \pm 0.11$ for RIXOS, where errors are $1 \sigma$ ). Since the slope of the EMSS $\log N-\log S$ curve is actually steeper than (although consistent with) that of RIXOS, there is no evidence from this comparison to support previous claims about incompleteness in the EMSS (e.g. Franceschini et al. 1994), in which incompleteness is thought to be a problem at low fluxes. The best-fitting $\mathrm{CF}$ is found to be $1.47 \pm 0.11$; again errors are $1 \sigma$. The standard CF of 1.8 is thus significantly different (rejected at $>99.0$ per cent) from that found by matching the $\log N-\log S$ curves. There is, however, no evidence from the RIXOS and EMSS $\log N-\log S$ relations that the CF should be as small as 1.0, a possibility considered by Boyle et al. (1994).

To assess the impact of the different CFs on AGN evolutionary models, values of 1.47 and 1.8 have both been used 


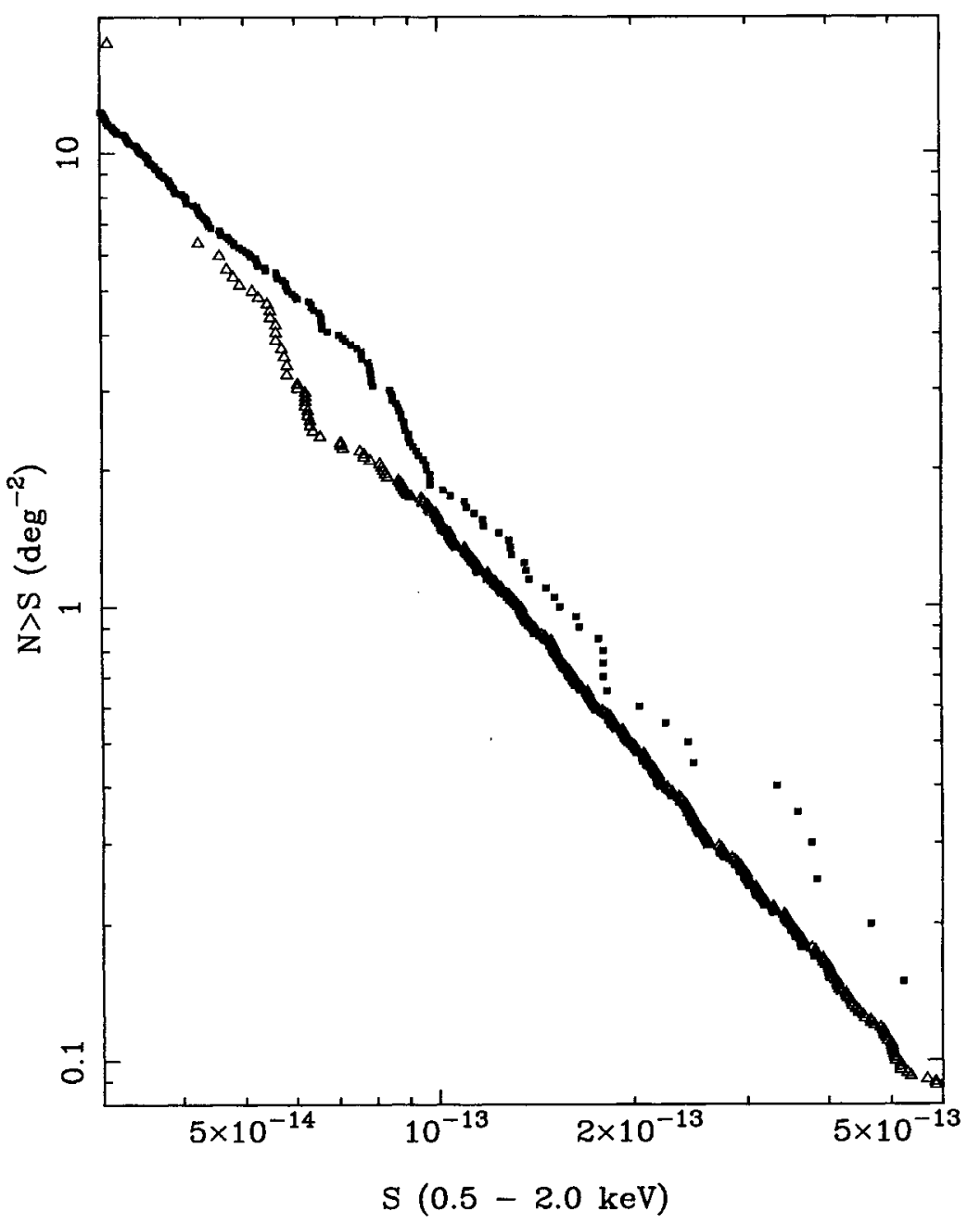

Figure 3. Integral $\log N-\log S$ relation of RIXOS AGN (filled squares) and EMSS AGN (open triangles).

in the subsequent analysis and the results compared. Note that all fluxes and luminosities quoted in this paper are for the $0.5-2.0 \mathrm{keV}$ band, i.e. the Einstein fluxes have been converted to the ROSAT flux band.

\section{THE LUMINOSITY FUNCTION}

The techniques that we have applied to the data are discussed in this section, while the results of the analysis are described in Section 5.

A flux-limited survey samples different luminosity ranges at different redshifts. The AGN population as a function of luminosity and redshift is represented by the $\mathrm{X}$-ray luminosity function (XLF), $\phi(L, z)$, which is defined as the number of objects detected per unit comoving volume per unit luminosity interval, i.e.

$\phi(L, z)=\frac{\mathrm{d}^{2} N}{\mathrm{~d} V \mathrm{~d} L}(L, z)$.

Evolution in the space density or luminosity of AGN with redshift is seen as a change in the XLF.

The two simplest forms for evolution are pure density and pure luminosity evolution (PLE). In pure density evolution, the number of AGN per unit comoving volume is assumed to evolve while the distribution of luminosities remains constant. In PLE, the number of AGN per unit comoving volume remains constant, while the luminosity of each AGN evolves.

Pure density evolution models do not predict the flattening at low fluxes which is seen in the best $\log N-\log S$ curves currently available, in both the optical (Boyle, Shanks \& Peterson 1988) and X-ray bands (Hasinger et al. 1993; Branduardi-Raymont et al. 1994). For this reason, recent evolutionary models have been based on luminosity evolution of some form. Only PLE models are considered in this paper.

As shown by Maccacaro et al. (1991), the XLF can be modelled as two power laws with a break luminosity, $L_{\text {break }}$, such that

$$
\begin{array}{ll}
\phi=K_{1} L^{-\gamma_{1}} & L<L_{\text {break }}, \\
\phi=K_{2} L^{-\gamma_{2}} & L>L_{\text {break }},
\end{array}
$$

where $K_{1}$ and $K_{2}$ are normalizations of the two power laws. Since we require the luminosity function to be continuous, the two normalizations are not independent. A single normalization $K_{1}$ is adequate, since 
$K_{2}=K_{1} / L_{\text {break }}^{\left(\gamma_{1}-\gamma_{2}\right)}$

In the framework of a PLE model, the XLF retains its shape at all redshifts, hence the XLF at any redshift depends on only the evolutionary law and the XLF at zero redshift (hereafter $z=0$ XLF).

\subsection{Maximum likelihood and two-dimensional Kolmogorov-Smirnov testing}

The maximum likelihood method (Marshall et al. 1984) has been used to obtain best-fitting evolutionary parameter(s) and the $z=0$ XLF for each evolution model, utilizing the full RIXOS + EMSS sample of AGN. This technique involves simultaneously fitting the evolution and the $z=0$ XLF. There are four or five free parameters [three from the $z=0 \operatorname{XLF}\left(\gamma_{1}, \gamma_{2}\right.$ and $\left.L_{\text {break }}\right)$, plus either one or two from the evolution model] in fitting the models considered in this paper. The normalization of the $z=0$ XLF is set so that the total number of objects predicted by the model is equal to the number in the sample, and is not a free parameter in the fit.

To test the acceptability of evolutionary models, the twodimensional Kolmogorov-Smirnov (2D KS) test has been used. Two alternatives have been used in the literature: the test described by Peacock (1983), and the test of Fasano \& Franceschini (1987), which is also described by Press et al. (1992). It is important to use the test most effective at rejecting poor models and accepting good models for the RIXOS and EMSS data sets, to ensure confidence in the results. To assess the most appropriate test to use, both were applied to simulated data as described in the next paragraph.

The two best-fitting XLFs and evolutionary models (see Section 5.1) from Maccacaro et al. (1991) were used to produce 200 simulated samples of AGN, 100 from each evolutionary model. Each sample was constructed using two completeness limits, appropriate to the RIXOS and EMSS samples respectively. The number of objects in each simulated sample was between 400 and 600 , similar to the number of objects in the combined RIXOS + EMSS sample, and the tests were performed over exactly the same plane as those used on the real sample (see below). In this way, the $2 \mathrm{D} \mathrm{KS}$ tests were evaluated under very similar conditions to those under which they were actually to be applied. Each sample was tested once against its own parent XLF and evolutionary model, and once against the other, so that 200 tests were performed with correct models and 200 with incorrect models. The Peacock test rejected 100 incorrect models ( 50 per cent) at the 95 per cent level and six correct models, while the Fasano \& Franceschini test rejected 84 incorrect models ( 42 per cent) and 10 correct models. This indicates that the 2D KS test of Peacock is more efficient at distinguishing between good and bad models of our data. Because the EMSS and RIXOS contain a very large range of sky coverage at different completeness limits, the combined RIXOS + EMSS sample has a slightly lower redshift-de-evolved luminosity correlation coefficient (typically 0.43 , but dependent on the cosmological and evolutionary model) than the simulation models (typically 0.5 to 0.7). According to Fasano \& Franceschini (1987), their test reaches maximum efficiency when compared to that of Peacock (1983) at higher correlation coefficients, and so it is reasonable to assume that the results obtained from our simulations should hold for the actual data to be tested. The test of Peacock (1983) has therefore been used in this paper.

Models have been tested in the redshift and de-evolved luminosity plane over a range that includes all parameter values found in RIXOS and the EMSS $(0<z<3.5$, $10^{40}<L_{0}<10^{47}$, where $L_{0}$ is the de-evolved $0.5-2.0 \mathrm{keV}$ luminosity in erg $\mathrm{s}^{-1}$; note that $L_{0}$ is model-dependent). Since there is no selection criterion in this analysis, based on observed X-ray luminosity, that would correspond to the $M_{B}<-23$ requirement often used in optical QSO surveys, the test has not been performed over an interval in observed luminosity. Imposing a lower limit to the observed luminosity in this way would introduce implicit density evolution to a PLE model (see Kassiola \& Mathez 1990), and is hence undesirable.

\section{$4.21 / V_{\mathrm{a}}$ and $\left\langle V_{\mathrm{e}} / V_{\mathrm{a}}\right\rangle$}

An estimate of the behaviour of the XLF can be gained in a model-independent way using the $1 / V_{\text {a }}$ statistic (Avni \& Bahcall 1980) and plotting the XLF in distinct redshift bins, as in Maccacaro et al. (1991). Of course, any evolution occurring within these distinct bins will not be apparent in this type of treatment.

A more quantitative treatment can be made using the $\left\langle V_{\mathrm{e}} / V_{\mathrm{a}}\right\rangle$ statistic (Avni \& Bahcall 1980). To investigate single-parameter PLE models, without simultaneously modelling the $z=0 \mathrm{XLF}$, we have carried out $\left\langle V_{\mathrm{e}} / V_{\mathrm{a}}\right\rangle$ tests in distinct redshift shells. In this case the test is used to obtain acceptable evolutionary parameters for the given model, and the bins have been chosen so that within each shell the evolutionary parameter has a 68 per cent confidence region of about \pm 20 per cent of its value over the entire redshift range; this is a good compromise between resolution in redshift and constraint of the evolutionary properties of each bin. The practical aspects of the $\left\langle V_{\mathrm{e}} / V_{\mathrm{a}}\right\rangle$ test within redshift shells are described by Della Ceca et al. (1992).

We have also used the $\left\langle V_{\mathrm{e}} / V_{\mathrm{a}}\right\rangle$ test to examine evolution at high redshift in a model-independent way. In this case the $\left\langle V_{\mathrm{e}} / V_{\mathrm{a}}\right\rangle$ test has been applied in the redshift interval $z_{\mathrm{b}}<z<3.5$ where $z_{\mathrm{b}}$ is varied between 1 and 3 . Here, the test is used with no evolutionary model, and is capable of determining whether the data are consistent with the no-evolution hypothesis in the range $z_{\mathrm{b}}<z<3.5$, and, if not, whether the luminosity function is increasing with redshift $\left(\left\langle V_{\mathrm{e}}\right\rangle\right.$ $\left.\left.V_{\mathrm{a}}\right\rangle>0.5\right)$ or decreasing with redshift $\left(\left\langle V_{\mathrm{e}} / V_{\mathrm{a}}\right\rangle<0.5\right)$. This use of the $\left\langle V_{\mathrm{e}} / V_{\mathrm{a}}\right\rangle$ test is described by Dunlop \& Peacock (1990).

\section{RESULTS}

The best-fitting evolutionary parameters and luminosity functions have been obtained from the combined RIXOS + EMSS sample. As a check for consistency between the two samples, they have been tested for goodness of fit both individually and in combination. Table 2 shows the results for the maximum likelihood and 2D KS tests applied to the models that have been investigated. Errors quoted were 
obtained using the method of Lampton, Margon \& Bowyer (1976), and correspond to $\Delta \chi^{2}=1$, i.e. 68 per cent confidence intervals for one interesting parameter.

\subsection{The simplest models}

The two simple PLE models used by Maccacaro et al. (1991) are power-law evolution,

$L=L_{0}(1+z)^{C}$,

and evolution that is exponential with look-back time,

$L=L_{0} \mathrm{e}^{C \tau}$,

where $C$ is the evolutionary parameter and $\tau$ is the lookback time:

$$
\begin{array}{ll}
\tau=z /(1+z) & q_{0}=0, \\
\tau=1-1 /(1+z)^{3 / 2} & q_{0}=0.5 .
\end{array}
$$

The 2D KS test rejects both of these simple models at the 95 per cent level for the combined sample, except for the $q_{0}=0$, $\mathrm{CF}=1.47$ case where the power-law model is just acceptable at the 95 per cent level. Note that the case where the 2D KS probability $P(>D)$ is 1.2 indicates that the difference between the expected and actual distributions is small, not that it is zero; it is possible for the test of Peacock (1983) to produce values for $P(>D)$ that are larger than 1, in which case it cannot be regarded as a probability, although the implication that a model is a good fit if $P(>D)$ is high is certainly true.

Figs 4 and 5 show binned $1 / V_{\mathrm{a}}$ luminosity functions in redshift shells against the best-fitting power-law and expo-

\begin{tabular}{|c|c|c|c|c|c|c|c|c|c|c|c|c|c|}
\hline model & $\mathrm{CF}$ & $q_{0}$ & $z_{\text {cut }}$ & C & $C_{1}$ & $K_{1}^{a}$ & $\gamma_{1}$ & $\gamma_{2}$ & $\begin{array}{c}\log _{10} \\
\left(L_{b r e a k}\right)^{c}\end{array}$ & $I_{X R B^{b}}$ & $\begin{array}{l}\mathrm{P}(>\mathrm{D}) \\
\text { RIXOS } \\
\text { +EMSS }\end{array}$ & $\begin{array}{l}\mathrm{P}(>\mathrm{D}) \\
\text { RIXOS }\end{array}$ & $\begin{array}{l}P(>D) \\
\text { EMSS }\end{array}$ \\
\hline$(1+z)^{C}$ & 1.8 & 0.0 & - & 2.66 & - & 2.18 & 1.68 & 3.38 & 3.59 & 8.24 & 0.020 & 0.26 & 0.036 \\
\hline$e^{C \tau}$ & 1.8 & 0.0 & - & 4.74 & - & 1.59 & 1.64 & 3.23 & 3.29 & 7.31 & 0.033 & 0.047 & 0.033 \\
\hline$(1+z)^{C}$ & 1.8 & 0.0 & 1.82 & 2.91 & - & 1.83 & 1.65 & 3.30 & 3.49 & 6.88 & 0.27 & 0.62 & 0.096 \\
\hline $10^{C z+C_{1} z^{2}}$ & 1.8 & 0.0 & - & 1.10 & -0.230 & 1.56 & 1.62 & 3.27 & 3.52 & 6.47 & 0.36 & 0.45 & 0.17 \\
\hline$(1+z)^{C}$ & 1.8 & 0.5 & - & 2.35 & - & 1.75 & 1.62 & 3.38 & 3.57 & 4.56 & 0.015 & 0.62 & 0.017 \\
\hline$e^{C \boldsymbol{r}}$ & 1.8 & 0.5 & - & 3.82 & - & 1.37 & 1.63 & 3.32 & 3.20 & 4.71 & 0.019 & 0.21 & 0.0048 \\
\hline$(1+z)^{C}$ & 1.8 & 0.5 & 1.42 & 2.94 & - & 1.22 & 1.57 & 3.30 & 3.38 & 4.18 & 0.69 & 0.24 & 0.25 \\
\hline $10^{C z+C_{1} z^{2}}$ & 1.8 & 0.5 & - & 1.07 & -0.238 & 1.48 & 1.60 & 3.33 & 3.46 & 4.20 & 0.58 & 0.58 & 0.21 \\
\hline$(1+z)^{C}$ & 1.47 & 0.0 & - & 2.46 & - & 2.00 & 1.64 & 3.23 & 3.64 & 8.28 & 0.055 & 0.55 & 0.042 \\
\hline$e^{C r}$ & 1.47 & 0.0 & - & 4.44 & - & 1.81 & 1.65 & 3.16 & 3.40 & 7.71 & 0.016 & 0.095 & 0.064 \\
\hline$(1+z)^{C}$ & 1.47 & 0.0 & 1.82 & 2.72 & - & 2.19 & 1.66 & 3.23 & 3.60 & 7.35 & 0.22 & 0.86 & 0.19 \\
\hline $10^{C z+C_{1} z^{2}}$ & 1.47 & 0.0 & - & 1.04 & -0.219 & 1.92 & 1.64 & 3.20 & 3.58 & 7.03 & 0.24 & 0.65 & 0.20 \\
\hline$(1+z)^{C}$ & 1.47 & 0.5 & - & 2.19 & - & 1.64 & 1.59 & 3.23 & 3.62 & 4.88 & 0.049 & 1.2 & 0.019 \\
\hline$e^{C \tau}$ & 1.47 & 0.5 & - & 3.47 & - & 1.52 & 1.61 & 3.19 & 3.31 & 4.86 & 0.026 & 0.24 & 0.045 \\
\hline$(1+z)^{C}$ & 1.47 & 0.5 & 1.41 & 2.73 & - & 1.39 & 1.57 & 3.19 & 3.48 & 4.57 & 0.22 & 0.38 & 0.14 \\
\hline $10^{C z+C_{1} x^{2}}$ & 1.47 & 0.5 & - & 1.04 & -0.238 & 1.54 & 1.58 & 3.22 & 3.51 & 4.69 & 0.38 & 0.80 & 0.18 \\
\hline \multicolumn{14}{|l|}{ \pm ERRORS } \\
\hline$(1+z)^{C}$ & & & & 0.08 & & & 0.08 & 0.08 & 0.10 & & & & \\
\hline$e^{C r}$ & & & & 0.20 & & & 0.07 & 0.08 & 0.05 & & & & \\
\hline$(1+z)^{C}$ & & & 0.10 & 0.10 & & & 0.07 & 0.08 & 0.05 & & & & \\
\hline $10^{C z+C_{1} z^{2}}$ & & & & 0.06 & 0.03 & & 0.10 & 0.09 & 0.06 & & & & \\
\hline
\end{tabular}

Table 2. Results of fitting evolutionary models.

${ }^{a} K_{1}$ in units of $10^{-4}\left(10^{40} \mathrm{erg} \mathrm{s}^{-1}\right)^{\left(\gamma_{1}-1\right)} \mathrm{Mpc}^{-3}$.

${ }^{b} I_{\mathrm{XRB}}$ in units of $10^{-9} \mathrm{erg} \mathrm{s}^{-1} \mathrm{~cm}^{-2} \mathrm{sr}^{-1}(1-2 \mathrm{keV})$.

${ }^{c} L_{\text {break }}$ in units of $10^{40} \mathrm{erg} \mathrm{s}^{-1}$. 
nential evolution model luminosity functions. Figs 6 and 7 show evolutionary parameters acceptable at 68 per cent for these two evolutionary models, derived from the $\left\langle V_{\mathrm{e}} / V_{\mathrm{a}}\right\rangle$ test in redshift intervals. These figures suggest that the exponential model fails because it requires unacceptably rapid evolution at low redshift (in Fig. 7 the low-redshift evolutionary parameter lies below any value that would be consistent with $z>0.4$ ), while the power-law model fails because it overpredicts evolution at high redshift (for $z>1.5$ the model curves in Fig. 4 lie well above the data). Evolution is slower at high redshift in the exponential model than in the power-law model, and hence in Fig. 5 the exponential model appears less discrepant at $z>1.5$ than the power-law model in Fig. 4. Figs 4 to 7 have been constructed using $q_{0}=0, \mathrm{CF}=1.8$.

\subsection{Two-parameter evolutionary models}

As both single-parameter models are rejected by the 2D KS test, more complex models have been investigated. From Figs 4 and 5 it appears that there is little difference in the slope of the luminosity function in different redshift bins, so models in which the luminosity function changes slope with redshift have not been considered. A power-law model with a redshift cut-off $z_{\mathrm{cu}}$, where

$$
\begin{array}{ll}
L=L_{0} \times(1+z)^{C} & z<z_{\text {cut }}, \\
L=L_{0} \times\left(1+z_{\text {cut }}\right)^{c} & z>z_{\text {cut }},
\end{array}
$$

and alternatively a polynomial evolution of the form

$L=L_{0} \times 10^{\left(C_{z}+C_{1} z^{2}\right)}$

both have two free parameters for the evolution (plus three for the luminosity function). Both of these models are accepted at the 95 per cent level by the 2D KS test for the combined RIXOS + EMSS samples for both values of $q_{0}$ and CF; there is little justification, from the 2D KS probabilities obtained, to prefer one of the models. The similarity of the two models for $z<1.5$ is illustrated by the predicted $N(z)$ relations plotted in Fig. 1 . The two evolutionary models are, however, radically different in shape beyond this redshift: in the polynomial evolutionary model, luminosity declines after $z \sim 2$, and at $z=3.5$ the expected number of objects differs by a factor of 5 for the two models. A larger sample of $z>2$ objects would have the potential to discriminate between the two models.

\subsection{Evolution at high redshift}

It has been seen in the previous section that PLE models in which evolution ceases or changes direction at high redshift

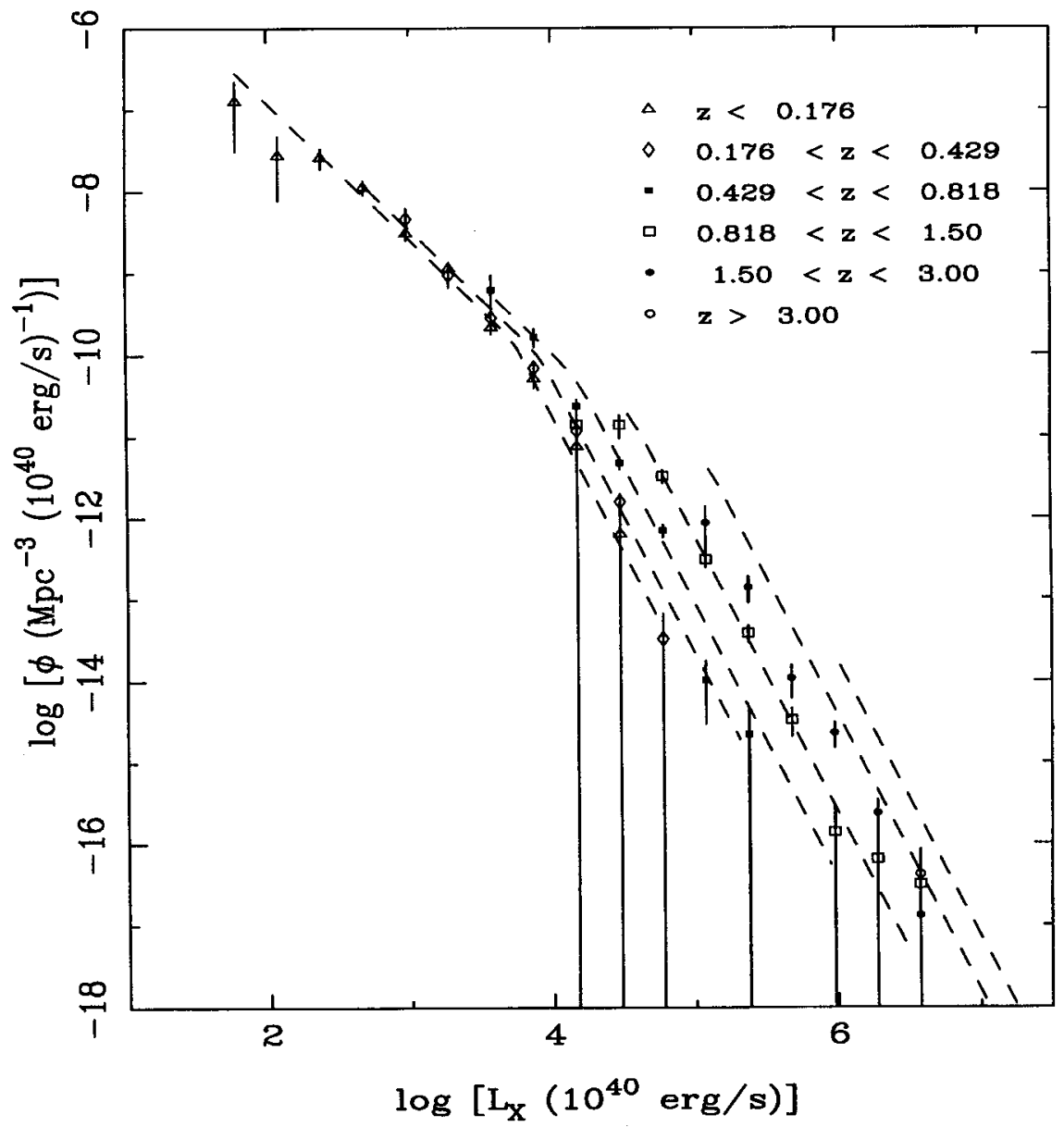

Figure 4. Binned $\left\langle 1 / V_{\mathrm{a}}\right\rangle$ XLF of the RIXOS + EMSS sample and best-fitting power-law evolution model XLF (dashed lines) for $q_{0}=0$ and $\mathrm{CF}=1.8$. 


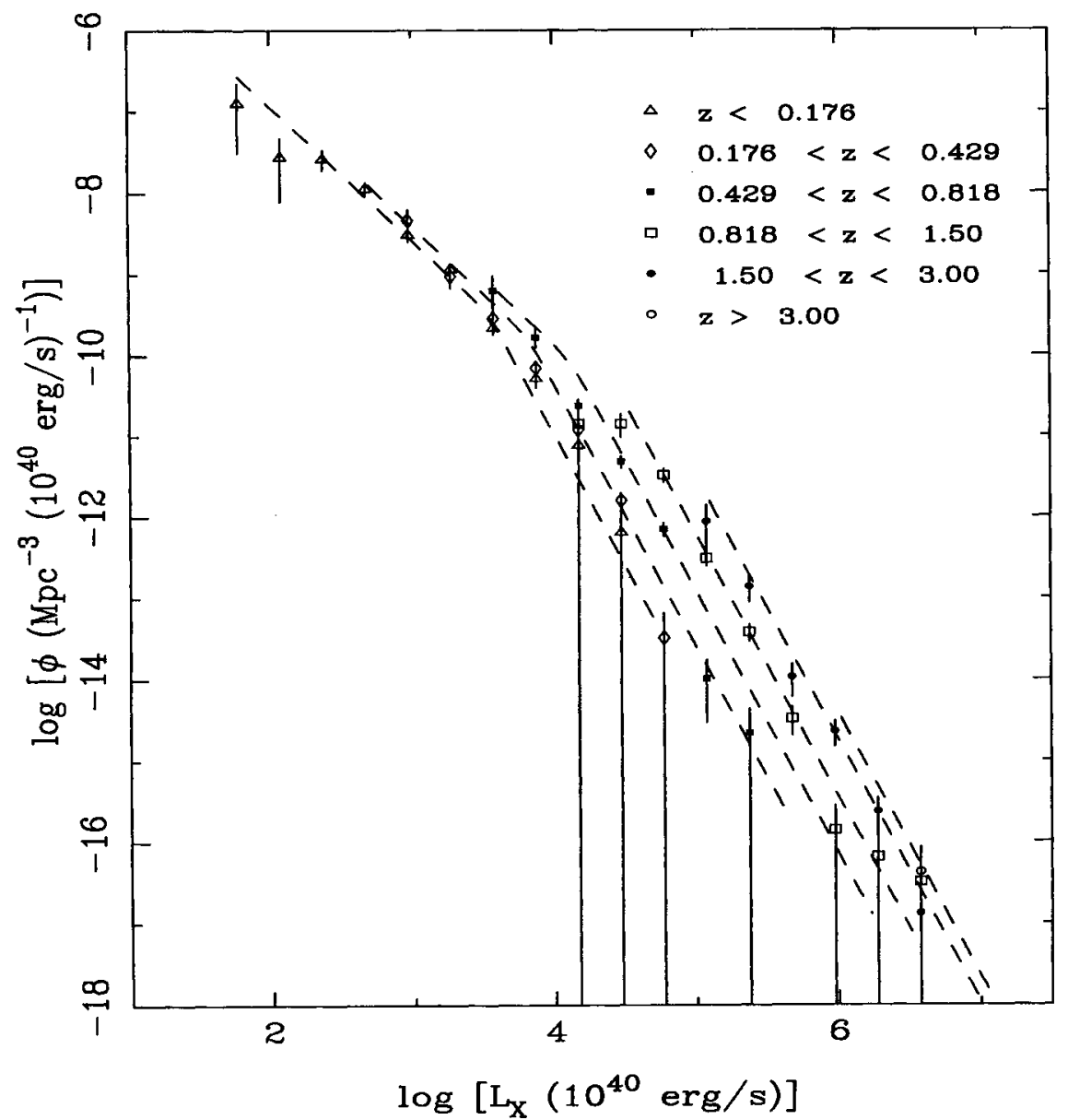

Figure 5. Binned $\left\langle 1 / V_{\mathrm{a}}\right\rangle$ XLF of the RIXOS + EMSS sample and best-fitting exponential evolution model XLF (dashed lines) for $q_{0}=0$ and $\mathrm{CF}=1.8$.

are found to be more acceptable than models where evolution continues, for both values of $q_{0}$ and $\mathrm{CF}$, indicating that evolution at $z>2$ must be absent or slow compared with that at low redshift. As further evidence, the results of the $\left\langle V_{\mathrm{e}} / V_{\mathrm{a}}\right\rangle$ test from $z=z_{\mathrm{b}}$ to $z=3.5$ for the combined RIXOS + EMSS sample are shown in Figs 8 and 9. Both were constructed using a CF of 1.8 ; when 1.47 is used, $\left\langle V_{\mathrm{e}} / V_{\mathrm{a}}\right\rangle$ is a few per cent lower; note that above $z_{\mathrm{b}}=2.4$ there are only four objects included in the test. From $z_{\mathrm{b}}=1.67$, with $30 \mathrm{AGN}$, the $\left\langle V_{\mathrm{e}} / V_{\mathrm{a}}\right\rangle$ test shows the data to be consistent at the 68 per cent level with no evolution for both values of $q_{0}$ and $\mathrm{CF}$. The $\left\langle V_{\mathrm{e}} / V_{\mathrm{a}}\right\rangle$ test used in this way is model-independent, and this result is not restricted to PLE models.

\section{DISCUSSION}

Although changing the ROSAT to Einstein CF from 1.8 to 1.47 does not appear to affect the choice of model (i.e. the power law with evolutionary cut-off and polynomial models are both significantly more acceptable than the simple power law and exponential models), it does have a significant effect on the best-fitting evolutionary parameter(s) and the $z=0 \mathrm{XLF}$. In all cases the best-fitting parameters for $\mathrm{CF}=1.47$ are outside the 90 per cent confidence region of the parameters for $\mathrm{CF}=1.8$; evolution is slower, and the steep part of the XLF is less steep if the CF of 1.47 is used.

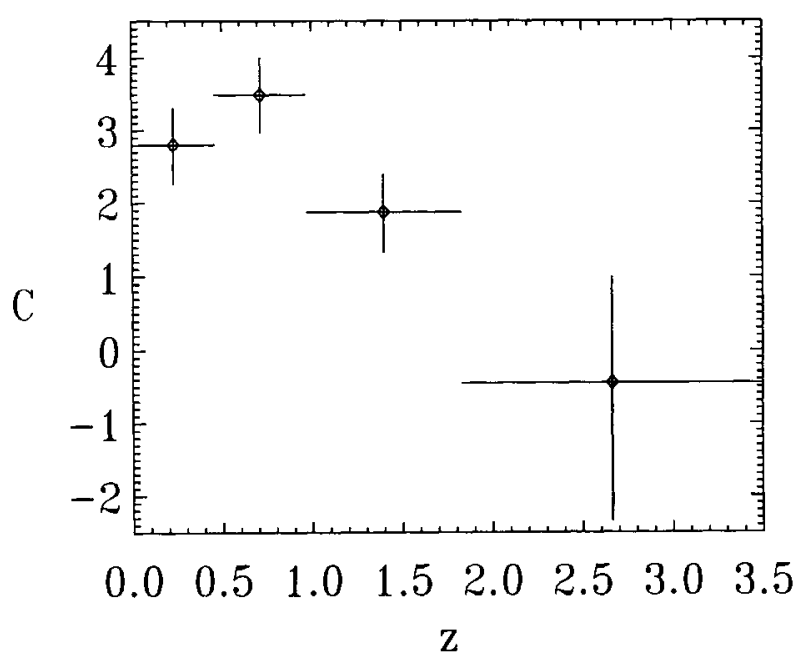

Figure 6. Power-law evolutionary parameter $C$ in redshift bins using the $\left\langle V_{\mathrm{e}} / V_{\mathrm{a}}\right\rangle$ test for $q_{0}=0$ and $\mathrm{CF}=1.8$.

The slope of the low-luminosity region of the XLF is affected little by the choice of CF.

The evolutionary parameter, $C$, for the exponential model is consistent at 68 per cent with the EMSS value $\left(C=4.18 \pm 0.35\right.$ for $\left.q_{0}=0\right)$ only for $\mathrm{CF}=1.47$, and its low $\mathrm{KS}$ probability confirms the result of Della Ceca et al. (1992) 
that this model is a poor description of AGN evolution. For the power-law model, the value of $C$ obtained here is consistent with that found by Maccacaro et al. (1991) $\left(2.56 \pm 0.17\right.$ for $\left.q_{0}=0\right)$ for both values of CF. The introduction of a cut-off in evolution at $z=1.8$ significantly improves the 2D KS probability of the power-law model for the RIXOS + EMSS sample; the last column of Table 2 shows that the EMSS data alone are also better fitted with the evolutionary cut-off. Including an evolutionary cut-off at $z=1.8$ in a $\left\langle V_{\mathrm{e}} / V_{\mathrm{a}}\right\rangle$ test to the EMSS data with a power-law evolutionary model and $q_{0}=0$ gives $C=2.74 \pm 0.20$, almost identical to the RIXOS + EMSS value for CF $=1.47$ and still consistent for $\mathrm{CF}=1.8$.

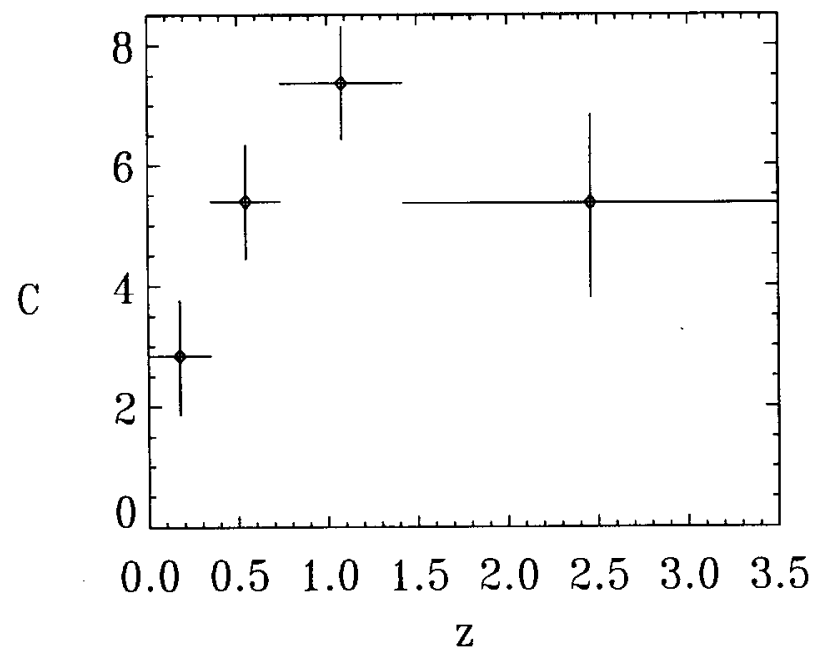

Figure 7. Exponential evolutionary parameter $C$ in redshift bins using the $\left\langle V_{\mathrm{e}} / V_{\mathrm{a}}\right\rangle$ test for $q_{0}=0$ and $\mathrm{CF}=1.8$.
Comparing the $\mathrm{CF}=1.8, q_{0}=0$ evolutionary parameters and $z=0$ XLFs from the RIXOS + EMSS sample with those of Boyle et al. (1994), we find similar values for all but the exponential evolution model which evolves faster in Boyle et al. (1994). For $q_{0}=0.5$, there is less agreement, with the $z=0$ XLFs of the exponential, power-law and polynomial evolution laws having significantly lower values for $\gamma_{1}$ (i.e. flatter slopes at low luminosity) in Boyle et al. (1994). The best-fitting $q_{0}=0.5$ power law with evolutionary cut-off model in Boyle et al. (1994), whilst having a similar $z=0$ XLF and evolution rate $C$, has a much higher cut-off redshift $\left(z_{\text {cut }}=1.7\right)$ than the value found here, $z_{\text {cut }}=1.4$ for $q_{0}=0.5$.

It is notable that almost all of the models considered in this paper are found to be more acceptable to the 2D KS test than in Boyle et al. (1994), in which almost all models are rejected at $>99$ per cent. Boyle et al. used the test of Fasano \& Franceschini (1987), while we have used that of Peacock (1983). However, we have also tested our models against the RIXOS + EMSS data set using the test of Fasano \& Franceschini, and do not find them to be rejected at $>99$ per cent. In Boyle et al. (1994), the inclusion of narrow-line objects gave significantly increased 2D KS probabilities (i.e. a better fit). However, narrow-line objects that would be classified by Stocke et al. as AGN have been included throughout this analysis. If we exclude these objects, which occur in both the EMSS and RIXOS, we do find lower 2D KS probabilities, although the polynomial and power law with evolutionary cut-off models are still acceptable at the 95 per cent level while the simple powerlaw and exponential evolution models are not. For the $z=0$ XLF, typically $\gamma_{1}$ is reduced by about 0.1 and $L_{\text {break }}$ is increased by 25 per cent to 50 per cent, depending on the specific PLE model and choice of cosmology; the best-

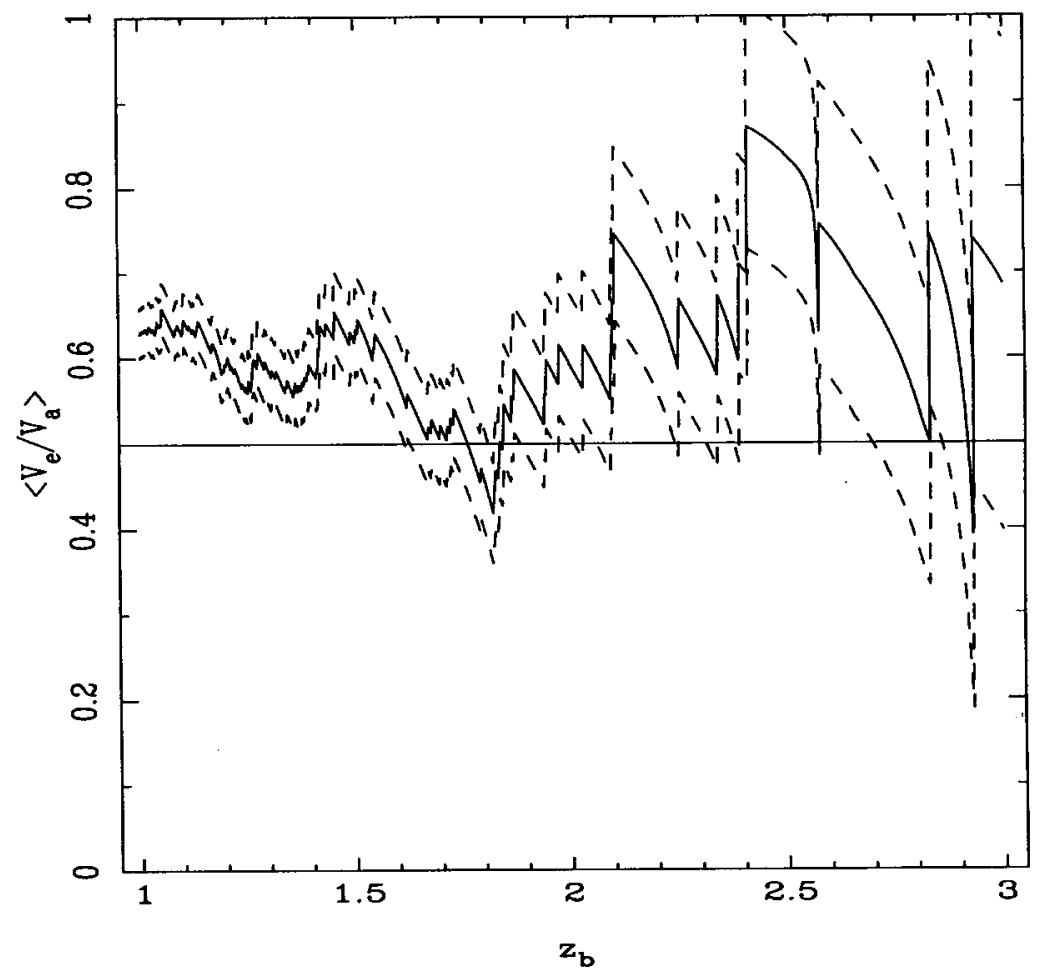

Figure 8. $\left\langle V_{\mathrm{e}} / V_{\mathrm{a}}\right\rangle$ test in the redshift interval $z=z_{b}$ to $z=3.5$ using the RIXOS + EMSS sample for $q_{0}=0$. 


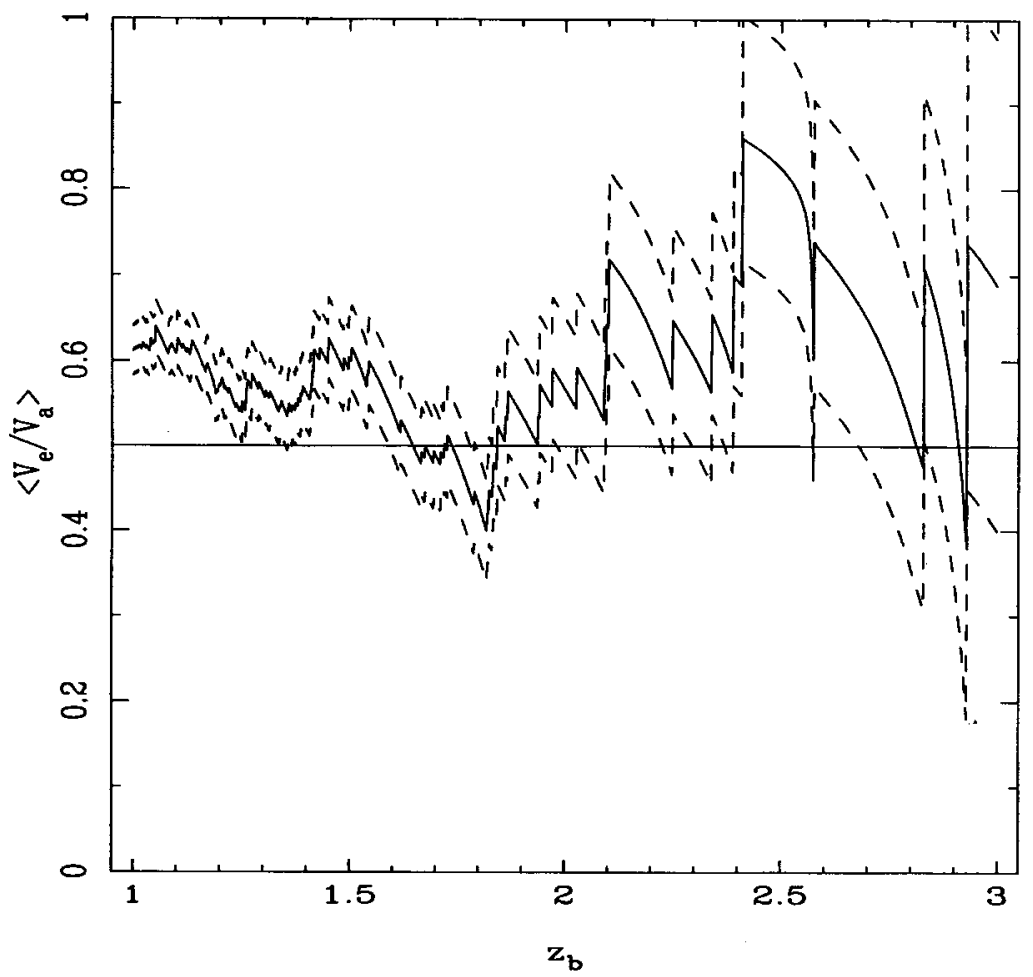

Figure 9. $\left\langle V_{\mathrm{e}} / V_{\mathrm{a}}\right\rangle$ test in the redshift interval $z=z_{\mathrm{b}}$ to $z=3.5$ using the RIXOS + EMSS sample for $q_{0}=0.5$.

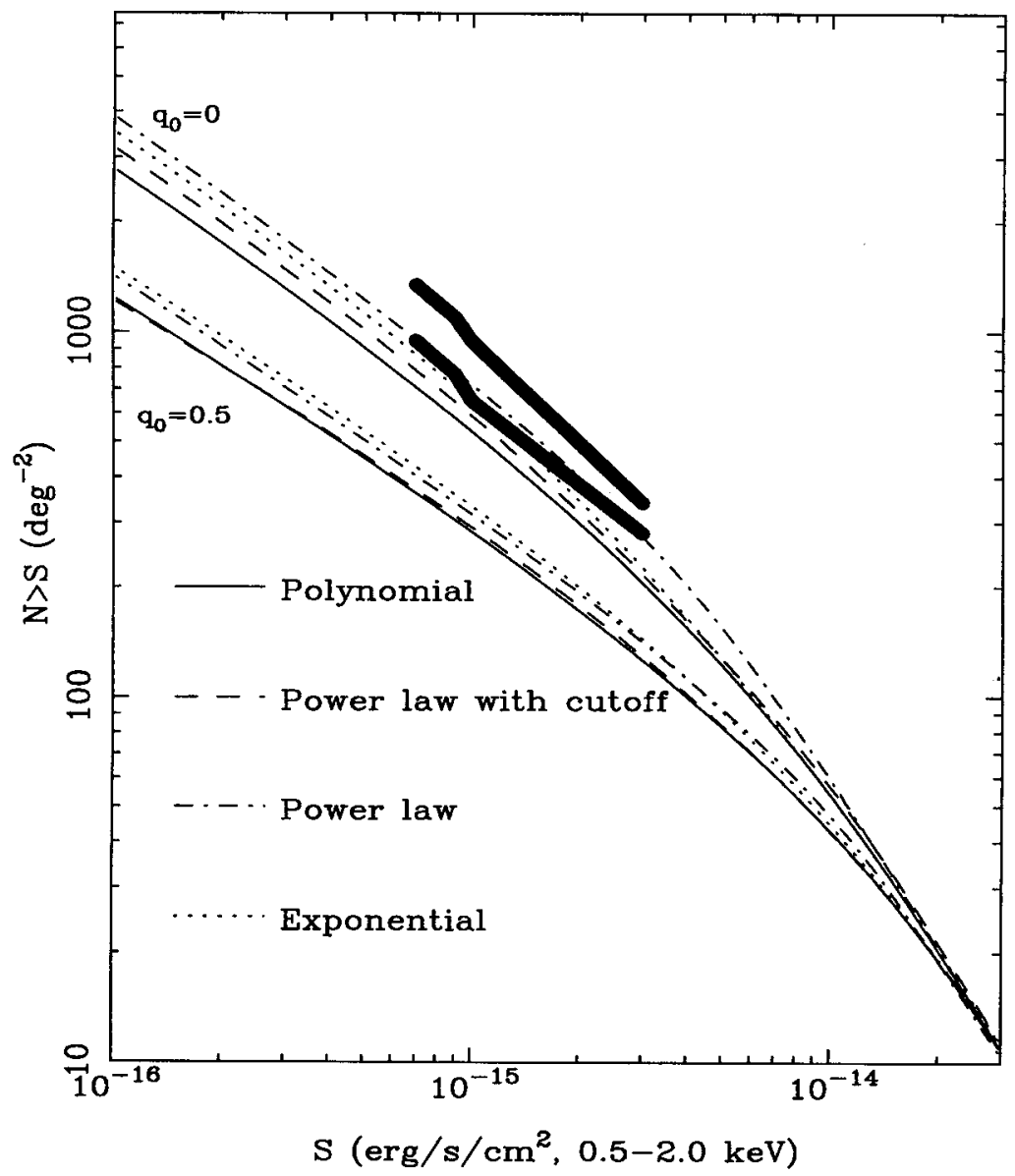

Figure 10. $\log N-\log S$ relation of the evolutionary models extrapolated to faint fluxes. The thick lines represent the $1 \sigma$ confidence limits to the total $\log N-\log S$ relation of all sources (i.e. not only AGN) at faint fluxes determined by fluctuation analysis of a deep ROSAT field by Barcons et al. (1994). 
fitting values of $\gamma_{2}$ and $z_{\text {cut }}$ change by no more than 0.02 and the evolutionary parameters remain within the errors quoted in Table 2 . There are no broad-line objects with $L_{0}<2 \times 10^{41} \mathrm{erg} \mathrm{s}^{-1}$.

The poor model fits of Boyle et al. (1994) may be attributable to the conversion between ROSAT and Einstein measured fluxes; the ROSAT sample of Boyle et al. (1994) combined with the EMSS AGN using a CF of 1.8 shows an evolutionary rate too high to be consistent with the EMSS sample alone. As we have discussed in Section 3, the effective CF from ROSAT to Einstein fluxes may be significantly lower than 1.8, and as seen in Table 2 a lower CF has the potential to reduce the evolutionary rate of combined ROSAT and Einstein data, and hence could improve the self-consistency and model fits of Boyle et al. (1994).

The $\log N-\log S$ relations at faint fluxes derived from the $\mathrm{CF}=1.8$ models tested above, and extended to $z=4$, are shown in Fig. 10. None of the model curves exceeds the total faint X-ray $\log N-\log S$ relation obtained by fluctuation analysis in Hasinger et al. (1993), or Barcons et al. (1994). Notably, the $\log N-\log S$ curves at faint fluxes for PLE are separated strongly by the value of $q_{0}$ used, while the specific choice of PLE model has a comparatively minor effect. In a $q_{0}=0.5$ universe, AGN undergoing PLE should represent between 30 and 45 per cent of all sources with $S>10^{-15}$ erg $\mathrm{s}^{-1} \mathrm{~cm}^{-2}$; in contrast, in a $q_{0}=0$ universe AGN evolving in this way would be expected to constitute between 55 and 100 per cent of these sources. Above $3 \times 10^{-14} \mathrm{erg} \mathrm{s}^{-1} \mathrm{~cm}^{-2}$, the $\log N-\log S$ curves from the PLE models are all very similar and represent the data well.

The AGN contribution to the $1-2 \mathrm{keV} X$-ray background has been calculated for $0<z<4,10^{40}<L_{0}<10^{47}$ (where $L_{0}$ is the de-evolved $0.5-2 \mathrm{keV}$ luminosity in $\mathrm{erg} \mathrm{s}^{-1}$ ) for all the models tested in Section 5, and is shown in Table 2 (column entitled $I_{\mathrm{XRB}}$ ). As expected from the $\log N-\log S$ predictions, the contribution of $A G N$ to the $\mathrm{X}$-ray background from these models has a stronger dependence on the value of $q_{0}$ than on the choice of PLE model. The values for the AGN X-ray background intensity given in Table 2 are in good agreement with those of Boyle et al. (1994). A recent measurement of the X-ray background (Chen, Fabian \& Gendreau 1996) using both $A S C A$ and ROSAT found an intensity of $1.46 \times 10^{-8} \mathrm{erg} \mathrm{s}^{-1} \mathrm{~cm}^{-2} \mathrm{sr}^{-1}(1-2 \mathrm{keV})$. Using this value, our acceptable models (power law with evolutionary cut-off and polynomial) predict that AGN account for between 44 and 50 per cent $\left(q_{0}=0\right)$ or 29 and 32 per cent ( $\left.q_{0}=0.5\right)$ of the 1-2 keV X-ray background, where these ranges include the uncertainty in $C F$. Taking the value of $1.25 \times 10^{-8} \mathrm{erg} \mathrm{s}^{-1} \mathrm{~cm}^{-2} \mathrm{sr}^{-1}$ for the $1-2 \mathrm{keV} \mathrm{X}$-ray background (Hasinger 1992) used by Boyle et al. (1994), the contribution from $A G N$ rises to between 52 and 60 per cent $\left(q_{0}=0\right)$ or 33 and 38 per cent $\left(q_{0}=0.5\right)$.

\section{CONCLUSIONS}

We have investigated the evolution of the XLF with redshift using a new sample of $198 \mathrm{X}$-ray-selected AGN with a spectroscopic completeness of 93 per cent at $3 \times 10^{-14} \mathrm{erg} \mathrm{s}^{-1}$ $\mathrm{cm}^{-2}$ ( 0.5 to $\left.2.0 \mathrm{keV}\right)$. We find PLE models, consistent with our data, in which the XLF declines or ceases to evolve beyond $z \sim 1.8$, and we find no evidence for evolution beyond this redshift from the model-independent $\left\langle V_{\mathrm{e}} / V_{\mathrm{a}}\right\rangle$ test. We have shown that narrow-emission-line galaxies at low redshift contaminating the AGN sample have little effect on the derived evolutionary properties. Furthermore, these conclusions are insensitive to the uncertainty in conversion of flux from the Einstein to the ROSAT passband, although this has a significant effect on the $z=0$ XLF and evolutionary rate for $z<1.8$.

\section{ACKNOWLEDGMENTS}

We thank all in the RIXOS team for their work in obtaining and reducing the data. The RIXOS project has been made possible by the award of International Time on the La Palma telescopes by the Comité Científico Internacional. This research has made use of data obtained from the UK ROSAT Data Archive Centre at the Department of Physics and Astronomy, University of Leicester (LEDAS), and we especially thank Mike Watson and Steve Sembay for their kind assistance. We thank the Royal Society for a grant to purchase equipment essential to the RIXOS project. KOM acknowledges the Royal Society for support and MJP acknowledges the support of a PPARC studentship through the course of this work. XB and IG-S are partially supported by the DGICYT under project PB92-0501.

\section{REFERENCES}

Avni Y., Bahcall J. N,, 1980, ApJ, 325, 694

Barcons X., Branduardi-Raymont G., Warwick R. S., Fabian A. C., Mason K. O., McHardy I. M., Rowan-Robinson M., 1994, MNRAS, 268, 833

Boyle B. J., Shanks T., Peterson B. A., 1988, MNRAS, 235, 935

Boyle B. J., Shanks T., Georgantopoulos I., Stewart G. C., Griffiths R. E., 1994, MNRAS, 271, 639

Boyle B. J., McMahon R. G., Wilkes B. J., Elvis M., 1995, MNRAS, 272,462

Branduardi-Raymont G. et al., 1994, MNRAS, 270, 947

Chen L.-W., Fabian A. C., Gendreau K. C., 1996, MNRAS, submitted

Della Ceca R., Maccacaro T., Gioia I. M., Wolter A., Stocke T. J., 1992, ApJ, 389, 491

Dunlop J. S., Peacock J. A., 1990, MNRAS, 247, 19

Fasano G., Franceschini A., 1987, MNRAS, 225, 155

Franceschini A., La Franca F., Cristiani S., Martin-Mirones J. M., 1994, MNRAS, 269, 683

Francis P. J., 1993, ApJ, 407, 519

Hasinger G., 1992, in Barcons X., Fabian A. C., eds, The X-ray Background. Cambridge Univ. Press, Cambridge, p. 229

Hasinger G., Burg R., Giacconi R., Hartner G., Schmidt M., Trumper J., Zamorani G., 1993, A\&A, 275, 1

Kassiola A., Mathez G., 1990, A\&A, 230, 255

Lampton M., Margon B., Bowyer S., 1976, ApJ, 208, 177

Maccacaro T., Gioia I. M., Wolter A., Zamorani G., Stocke J. T., 1988, ApJ, 326, 680

Maccacaro T., Della Ceca R., Gioia I. M., Morris S. L., Stocke J. T., Wolter A., 1991, ApJ, 374, 117

Marshall H. L., Avni Y., Braccesi A., Huchra J., Tananbaum H., Zamorani G., Zitelli V., 1984, ApJ, 283, 50

Peacock J. A., 1983, MNRAS, 202, 615

Press W. H., Teukolsky S. A., Vetterling W. T., Flannery B. P., 1992, Numerical Recipes in Fortran. Cambridge Univ. Press, Cambridge, p. 640

Primini F. A., Murray S. S., Huchra J., Schild R., Burgh R., Giacconi R., 1991, ApJ, 374, 440

Stocke J. T., Morris S. L., Gioia I. M., Maccacaro T., Schild R., Wolter A., Fleming T. A., Henry J. P., 1991, ApJS, 76, 813 